\title{
Chronic Thromboembolic Pulmonary Hypertension
}

\author{
Lara M. Wittine, MD \\ William R. Auger, MD*
}

\author{
Address \\ *Division of Pulmonary and Critical Care Medicine, University of California, \\ San Diego, 9300 Campus Point Drive, La Jolla, CA 92037, USA \\ Email: bauger@UCSD.edu \\ Published online: 12 March 2010 \\ (C) The Author(s) 2010. This article is published with open access at Springerlink.com
}

\section{Opinion statement}

The pulmonary hypertension $(\mathrm{PH})$ and right heart dysfunction that results from chronic thromboembolic involvement of the pulmonary vascular bed is potentially curable with surgical endarterectomy. Over the past several decades, growing clinical experience has brought about increased recognition of this treatable form of PH. Moreover, advances in cardiothoracic surgical techniques have given an increasing number of patients with chronic thromboembolic PH (CTEPH) a surgical remedy with decreasing perioperative morbidity and mortality risks. The availability of pulmonary hypertensive-specific medical therapy for CTEPH patients with surgically inaccessible disease also has been a positive therapeutic advance over the past several years. However, despite this progress, chronic thromboembolic disease as a sequela of acute pulmonary emboli continues to be underappreciated. Furthermore, even if CTEPH has been appropriately diagnosed, misinterpretation of diagnostic information may lead to the inappropriate exclusion of patients from surgical consideration. This may result in the prescription of pulmonary hypertensive medical therapy in CTEPH patients with potentially surgically correctable disease. This difficulty arises from a lack of objective criteria as to what constitutes surgical chronic thromboembolic disease, which primarily is a result of the variability in surgical experience in specialty centers in the United States. Consequently, clinicians must be wary about using pulmonary hypertensive medications in CTEPH patients. Before prescription, it is important to exclude patients from surgical consideration by consulting a specialized center with expertise in this discipline.

\section{Introduction}

Chronic thromboembolic residua may occur following a single or recurrent episode of acute pulmonary embolism. In patients with pulmonary embolism, the mechanical obstruction of the pulmonary vascular bed, along with the gradual development of a small vessel vasculopathy in the unobstructed vascular bed, may result in pulmonary hypertension $(\mathrm{PH})[1 \bullet]$. Estimates of the incidence of chronic thromboembolic $\mathrm{PH}$ 
(CTEPH) after acute pulmonary embolism range from $0.5 \%$ to $3.8 \%[2,3]$. If unrecognized or left untreated, progressive right ventricular dysfunction with the ultimate development of right heart failure is the expected outcome. Additional observations relevant to the diagnosis of CTEPH include the following: There appears to be no age or gender bias for the diagnosis of CTEPH; this disease has been observed in pediatric patients [4]. A history of acute venous thromboembolism is not present in approximately $30 \%$ of patients presenting with CTEPH [1]. Factors that appear to predispose to the development of CTEPH include recurrent embolic events, elevated pulmonary pressures at presentation of an acute pulmonary embolic event, and greater than $50 \%$ occlusion of the pulmonary vascular bed after a "single" embolic occurrence $[2,5]$. Thrombophilic states associated with CTEPH include the presence of a lupus anticoagulant, elevated levels of antiphospholipid antibodies, and elevated levels of factor VIII [6]. Deficiencies of protein C, protein S, and antithrombin III, or the presence of factor V Leiden and factor II mutations, do not appear to be associated with a higher risk of CTEPH.

The symptoms and signs of CTEPH are similar to those of other forms of $\mathrm{PH}$ and depend on the severity of the disease at presentation (Table 1). Exertional dyspnea and/or an unexplained decline in functional status are the most frequent presenting complaints. With the development of a significant degree of right ventricular dysfunction, symptoms such as exertional presyncope and physical signs including peripheral edema, jugular venous distention, and hepatomegaly may become evident. A unique physical finding in $30 \%$ of patients with CTEPH is the presence of flow bruits over the lung fields, a finding not encountered in patients with small vessel variants of pulmonary arterial hypertension (PAH).

Unlike other variants of PAH, CTEPH is potentially amenable to surgical correction. Although vessel obstructions with chronic thromboemboli may occur at any level in the pulmonary arterial system, only those involving the main, lobar, or segmental arteries are amenable to surgical correction. Therefore, the primary purpose of the diagnostic evaluation is to quantify the degree of $\mathrm{PH}$ and right heart dysfunction (ie, right heart catheterization), to establish its etiology, and to determine the proximal extent of disease (Fig. 1) $[7 \bullet \bullet]$.

The preferred therapy for appropriate patients with CTEPH is surgical removal of the chronic thromboembolic lesions within the proximal vessel with the intent to restore normal cardiopulmonary hemodynamics, improve functional status, and positively affect survivorship [8]. However, medical therapies directed at treating $\mathrm{PH}$ have been investigated and increasingly used in subcategories of CTEPH patients [9, 10]: 1) patients deemed inoperable because of extensive distal precapillary vasculopathy not amenable to surgery or those who have significant comorbidities that contraindicate surgery $[11]$; 2) the $10 \%$ to $15 \%$ of patients who have residual PH following thromboendarterectomy surgery [12]; and 3) patients with severe hemodynamic compromise, as a bridge to surgery. 


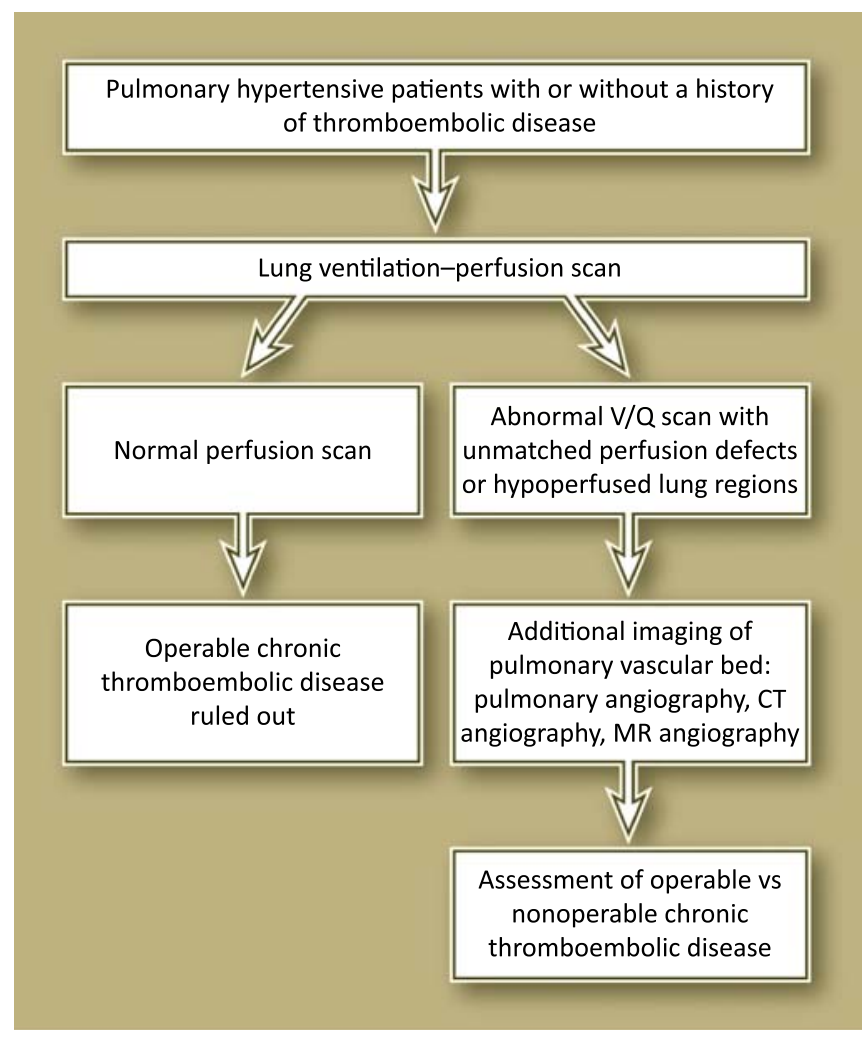

Figure 1. Diagnostic approach to chronic thromboembolic pulmonary hypertension. MR-magnetic resonance; $\mathrm{V} / \mathrm{Q}-$

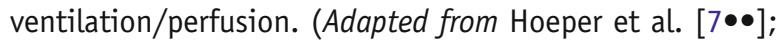
with permission.)

\section{Treatment}

\section{Pharmacologic treatment}

\section{Anticoagulation}

- The mainstay of therapy for patients with CTEPH is anticoagulation, regardless of candidacy for surgical endarterectomy. Whether or not a thrombophilic state has been identified, lifelong anticoagulation is recommended $[1,7 \bullet \bullet]$. The most commonly used anticoagulant is warfarin, usually with an international normalized ratio (INR) goal of 2.5 to 3.5. However, this INR target often is modified on an individual basis, such as in patients with comorbidities necessitating the use of medications that might increase bleeding risks (eg, aspirin products), elderly patients (a lower INR target), and individuals with an underlying thrombophilia (eg, those with an antiphospholipid syndrome, in whom a higher INR target might be recommended). The most common side effect of anticoagulation is an excessive bleeding tendency. For patients with contraindications to warfarin therapy, or those who have failed to adequately prevent thrombosis with warfarin, an alternative anticoagulant regimen should be administered. The use of low molecular weight heparins or factor 
Xa inhibitors (fondaparinux) may be considered. Warfarin also is contraindicated during pregnancy, necessitating transition to a low molecular weight heparin or another acceptable anticoagulant regimen for postendarterectomy patients who choose and are clinically able to become pregnant.

\section{Pulmonary hypertension pharmacotherapy}

- The histopathologic examination of distal arteries in CTEPH patients reveals vascular changes similar to those in patients with primary or idiopathic PAH [13]. The mechanism as to why proximal obstruction might lead to precapillary arteriography is not known. However, this observation, as well as the acknowledgment that PH in CTEPH patients does not entirely result from mechanical obstruction of the pulmonary vasculature, establishes a pathophysiologic context for the use of pulmonary vasodilator therapy in this patient population.

- Pharmacologic therapy for CTEPH has focused on three main classes of drugs currently prescribed for treating idiopathic PAH: endothelin receptor antagonists (ERAs), cyclic guanosine monophosphate (cGMP) phosphodiesterase 5 (PDE5) inhibitors, and prostanoids. Several open-label studies of pharmacologic agents performed in small patient groups suggest a role for medical therapy in those with inoperable or residual postoperative CTEPH [14-19]. However, as few well-designed placebo-controlled, randomized control trials have been performed to evaluate the efficacy of drug treatment in these patients, it is difficult to make a strong recommendation regarding appropriate therapeutic choice.

- The BENEFiT (Bosentan Effects in Inoperable Forms of Chronic Thromboembolic Pulmonary Hypertension) trial was a doubleblind, randomized, placebo-controlled study examining the use of the ERA bosentan in patients with inoperable CTEPH or residual/ recurrent $\mathrm{PH}$ post endarterectomy. This study of 57 patients showed improved pulmonary hemodynamics (pulmonary vascular resistance [PVR] $-24.1 \%$ of baseline) after 4 months of treatment with bosentan $[20 \bullet \bullet]$. The hemodynamic change, however, did not render a significant improvement in exercise capacity, and it is speculated that the trial did not continue long enough to be able to evaluate for this end point adequately. Sildenafil, a cGMP PDE5 inhibitor, also has been investigated as a treatment for inoperable CTEPH. The one randomized, placebo-controlled study of sildenafil use in patients with inoperable CTEPH was too underpowered ( $N=19$ enrolled patients) to prove functional benefit, but the open-label portion of this study suggested that prolonged (1-year) therapy with this medication may improve PVR, exercise capacity, and symptom scores [21]. Although changes in pulmonary hemodynamics have been seen to occur with short-term administration (3-4 months) of bosentan and sildenafil, longer followup is needed to determine whether this will translate into improved 
functional status and exercise capacity in those not able to fully benefit from endarterectomy $[20 \bullet \bullet, 21]$. According to the recommendations from the Fourth World Symposium on Pulmonary Hypertension for CTEPH patients with inoperable or residual postoperative disease, these patients should be referred to medical centers experienced in treating such patients for possible inclusion in clinical trials to investigate pharmacologic treatments $[7 \bullet \bullet]$.

- In CTEPH patients who are surgical candidates for thromboendarterectomy, a variety of pharmacologic agents, including bosentan, prostacyclins, and iloprost, have been studied as a bridge to pulmonary endarterectomy (PEA); however, overall, no significant difference in postoperative hemodynamics was detected in patients who received drug before endarterectomy compared with those undergoing surgery alone $[22,23]$. A large retrospective study confirmed these findings and raised the concern that patients receiving drug therapy have delayed referral to surgery and increased medical costs [10]. However, much of the impetus to affect pulmonary hemodynamics before surgery comes from the observation that CTEPH patients with preoperative PVR greater than 1,000 dynes $\cdot \mathrm{s} \cdot \mathrm{cm}^{-5}$ have a significantly higher perioperative mortality risk [4]. An open-label study investigating continuous intravenous (IV) prostacyclin in 12 patients with severe PH (PVR $>1200$ dynes $\cdot \mathrm{s} \cdot \mathrm{cm}^{-5}$ ) undergoing endarterectomy surgery demonstrated a significant reduction in preoperative PVR, with a trend toward improved operative mortality [22]. Because no definitive evidence exists that pulmonary vasodilator therapy positively affects outcomes in severe, hemodynamically compromised surgical candidates (PVR $>1000$ dynes $\left.\cdot \mathrm{s} \cdot \mathrm{cm}^{-5}\right)$, the preoperative administration of prostanoids, ERAs, and/or PDE5 inhibitors in this patient group should be coordinated with a center specializing in the surgical management of CTEPH.

Endothelin 1 is a potent vasoconstrictor that appears in higher levels in patients with $\mathrm{PH}$ and affects the vasculature via endothelin receptors A and B. ERAs block pulmonary vasoconstriction via one receptor (selective) or both receptors (nonselective).

Bosentan, a nonselective ERA, is the most thoroughly and rigorously studied to date of all pharmacologic therapies (via the BENEFiT trial) for patients with inoperable CTEPH or post-PEA residual PH. Bosentan improves cardiopulmonary hemodynamics, as evidenced by significant decreases in PVR and significantly improved cardiac indices. Decreased levels of pro-brain natriuretic peptide (pro-BNP) also suggest reduced right ventricular strain $[20 \bullet \bullet]$.

Standard dosage Bosentan: initial oral dose of $62.5 \mathrm{mg}$ twice a day for 4 weeks, increasing to target dose of $125 \mathrm{mg}$ twice a day. Laboratory monitoring: monthly liver function tests and complete blood count at 1 month, 3 months, then quarterly. If applicable, pregnancy screening should be done monthly and if there is an immediate concern about potential pregnancy $[20 \bullet \bullet, 24]$. 
Contraindications Pregnancy (bosentan is a known teratogen), hypersensitivity, and concomitant administration of cyclosporine, glyburide, and lopinavir/ ritonavir [24].

Main drug interactions Contraindicated: cyclosporine and glyburide. Major interaction: ritonavir [24].

Main side effects Mild liver function abnormalities (7-11\%) to fulminant liver failure (rare), peripheral edema (5-14\%), palpitations (5\%), flushing (7-14\%), headache

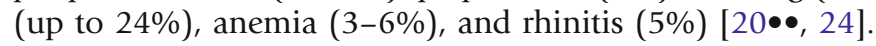

Phosphodiesterase 5 inhibitors

Standard dosage

Contraindications

Main drug interactions

Main side effects
$\mathrm{PH}$ is associated with upregulation of vascular PDE5, which rapidly degrades cGMP, leading to reduced levels of nitric oxide, a potent vasodilator. PDE5 inhibitors enhance nitric oxide-mediated vasodilation. They also may have additional beneficial effects on platelet activation and pulmonary vascular remodeling.

Sildenafil, a selective PDE5 inhibitor, significantly decreases PVR within 3 months of starting therapy in patients deemed inoperable or with residual PH after PEA surgery. This initial improvement translates into significantly improved hemodynamics (including cardiac index), BNP level, 6-min walk distance, and symptom scores with 12 months of therapy, possibly suggesting that sildenafil has disease-modifying effects on the pulmonary vasculature [21].

Sildenafil: oral administration starting at $20 \mathrm{mg}$ three times daily.

Concomitant use of nitrates (eg, nitroglycerin); hypersensitivity [24].

Contraindicated: erythrityl tetranitrate, isosorbide di- or mononitrate, nitroglycerin, and pentaerythritol tetranitrate. Major interaction: dihydrocodeine and ritonavir [24].

Flushing (10\%), dyspepsia (up to 30\%), headache (16-46\%), visual disturbance $(3-11 \%)[21,24]$.

\section{Prostanoid therapy}

Prostanoids have several important effects on the vasculature; they are potent vasodilators that also inhibit vascular smooth muscle proliferation and platelet aggregation.

Continuous IV administration of epoprostenol via infusion pump to patients with PVR greater than 1200 dynes $\cdot \mathrm{s} \cdot \mathrm{cm}^{-5}$ before PEA surgery may significantly increase preoperative cardiac output and reduces PVR $[10,22]$. These effects translate into reduced right ventricular strain, as measured by decreased preoperative BNP levels [22].

Open-label trials have shown that continuous subcutaneous administration of treprostinil improves hemodynamics, functional capacity, and survivability in patients with inoperable CTEPH [18]. An inhaled prostanoid, iloprost, also has been studied with variable results in CTEPH patients [19, 23]. One small study demonstrated improved pulmonary hemodynamics with acute administration of inhaled iloprost in 20 CTEPH patients (14 inoperable), suggesting a component of pulmonary vasoreactivity in these patients. However, sustained benefit with this therapy was not examined [19]. 
Standard dosage Epoprostenol: $2 \mathrm{ng} / \mathrm{kg} / \mathrm{min}$ IV initially, increasing in increments of $1 \mathrm{ng} / \mathrm{kg} /$ min to tolerance with a continuous infusion pump [22]. Treprostinil: subcutaneous administration beginning at $2 \mathrm{ng} / \mathrm{kg} / \mathrm{min}$, increasing to tolerance with a continuous infusion pump. The infusion site should be changed about every 28 days [18]. Continuous IV formulations also are available. Iloprost: $5-\mu$ inhalation via nebulizer up to six times a day [19].

Contraindications Severe left ventricular systolic dysfunction, hypersensitivity, and pulmonary edema [24].

Main drug interactions Major interaction: citalopram, clovoxamine, desvenlafaxine, duloxetine, escitalopram, femoxetine, flesinoxan, fluoxetine, fluvoxamine, milnacipran, nefazodone, paroxetine, sertraline, venlafaxine, and zimeldine [24].

Main side effects Flushing (up to 60\%), jaw pain (54-75\%), headaches (46-83\%), epigastric discomfort (up to 30\%), loose stools (up to 50\%), cough (with iloprost; up to $39 \%$ ), and bronchospasm (with iloprost) $[22,24]$.

\section{Future directions}

- Without surgical intervention, the prognosis for CTEPH patients previously was observed to be poor $[1 \bullet]$. However, preliminary reports suggesting hemodynamic and functional benefit from the use of PH-specific pharmacotherapy in CTEPH patients who are not surgical candidates have been encouraging, with evidence of improved quality of life and survivorship in this patient group [25•]. Well-powered, randomized, placebo-controlled trials are needed to evaluate $\mathrm{PH}$ pharmacotherapy in these patients, and as in $\mathrm{PAH}$, investigation of combination therapy likely is on the horizon.

- The routine placement of inferior vena cava filters in patients with CTEPH is not a universally accepted practice. The proposed benefit is to reduce the risk of perioperative and/or recurrent pulmonary embolism in patients with diagnosed CTEPH. At the University of California, San Diego (UCSD), inferior vena cava filter placement, in addition to lifelong anticoagulation, has resulted in recurrent, operable CTEPH in fewer than $1 \%$ of patients who have undergone thromboendarterectomy surgery.

- Pulmonary thromboendarterectomy, or what is increasingly referred to as pulmonary endarterectomy surgery, has become the preferred treatment for selected patients with CTEPH. Often, it is permanently curative, as long as patients remain on lifelong anticoagulation, and may result in a substantial improvement in cardiopulmonary hemodynamics [8, 12, 26-28]. Significant improvements in New York Heart Association functional class, dyspnea scores, gas exchange/exercise capacity, and long-term survival have been reported [25•, 29-31]. 


\section{Surgical candidacy}

- Important determinants of surgical candidacy for PEA are 1) the presence of chronic organized thrombi involving the main, lobar, and/or proximal segmental pulmonary arteries; 2) hemodynamic impairment proportional to the anatomically evident disease; and 3) the lack of other significant comorbid conditions that would contraindicate surgery $[1 \bullet, 26]$. Although patients with PVR greater than 1000 dynes $\cdot \mathrm{s} \cdot \mathrm{cm}^{-5}$ have increased perioperative mortality $[4,27]$, referral to centers specialized in surgical treatment of CTEPH should still be pursued in a timely manner. In these cases, preoperative pharmacologic treatment to reduce PVR should be considered by the medical-surgical endarterectomy team [26].

\section{PEA surgery}

- The management of CTEPH patients involves a multidisciplinary team of surgeons, intensivists, pulmonologists, and other specialists who aid in determining surgical candidacy and caring for the patient in the perioperative setting. The surgery involves a median sternotomy followed by the initiation of cardiopulmonary bypass. The patient is cooled to the point of hypothermia; periods of intermittent circulatory arrest allow adequate visualization of the pulmonary vasculature so consecutive bilateral thromboendarterectomies can be performed [4]. PEA involves careful circumferential dissection of intraluminal fibrinous material away from the arterial intima [4, 12]. After the specimens are removed, the patient is warmed and the surgical sites are sutured closed. Occasionally, a pericardial patch may be required to close the pulmonary artery and restore its original dimensions.

\section{Postoperative complications}

- Although not inclusive, the list of common post-PEA complications is similar to that of other open chest procedures and occurs at a similar frequency: mediastinal hemorrhage, ventilator-associated pneumonia, wound infections, atrial arrhythmias, delirium, and phrenic nerve injury $[8,27]$. For patients with antiphospholipid syndrome, as well as CTEPH patients undergoing an endarterectomy of a main pulmonary artery, early postoperative rethrombosis is of particular concern. The experience at UCSD has resulted in the practice of therapeutic anticoagulation within hours following the endarterectomy. Late development (1-3 weeks postoperative) of an inflammatory pericarditis occasionally may result in hemodynamic compromise and requires percutaneous or surgical drainage. Relatively unique to this patient population is the risk of postendarterectomy reperfusion lung injury (RPLI), an acute high-permeability, inflammatory condition that may develop within the first $24 \mathrm{~h}$ of surgery and is associated with increased oxygen and ventilator requirements. Postendarterectomy RPLI can range from mild to severe acute lung injury (occasionally 
manifesting as pulmonary hemorrhage) that may result in death $[1,8,27]$. RPLI usually is treated with supportive care, but in severe cases, inhaled nitric oxide and even extracorporeal life support may be required [32]. Another postoperative difficulty is residual PH (defined as postsurgical PVR $>500$ dynes $\cdot \mathrm{s} \cdot \mathrm{cm}^{-5}$ ) despite PEA surgery, which is reported in $10-15 \%$ of patients $[27,33]$. Although the presence of $\mathrm{PH}$ after PEA does not typically mean there has been no hemodynamic benefit from surgery, the presence of severe $\mathrm{PH}$ and right ventricular failure postoperatively is associated with a much greater mortality risk. In an analysis of 500 patients who underwent PEA, 17 of the 22 patients who died exhibited residual high pulmonary pressures [4].

\section{Mortality}

- With improvements in surgical technique, increased experience with this patient population, and a multidisciplinary approach to perioperative care, operative mortality has been reduced to $4-7 \%$ at select centers of excellence $[4,26]$. Because the early mortality statistics were as high as $17-23 \%[4,26]$, it is imperative to refer CTEPH patients to centers with considerable experience in the medical and surgical management of this disease.

\section{Other considerations}

- In patients with risk factors for coronary atherosclerosis, coronary angiography is recommended before surgery to determine whether cardiac artery bypass grafting should also be performed at the time of PEA. Valve repair or replacement, if required, also may be done during thromboendarterectomy [34]. Although tricuspid regurgitation is common in CTEPH patients, postoperative remodeling of the heart following relief of high pulmonary pressures may result in renewed tricuspid valve competence [4]. Therefore, tricuspid valve repair rarely is required and is performed only in patients with a severely damaged valve. Lastly, if there is evidence of improvement in the pulmonary hemodynamics as the organized thrombi are removed, surgical closure of a patent foramen ovale may be performed.

\section{Disclosure}

No potential conflicts of interest relevant to this article were reported.

\section{Open Access}

This article is distributed under the terms of the Creative Commons Attribution Noncommercial License which permits any noncommercial use, distribution, and reproduction in any medium, provided the original author(s) and source are credited. 


\section{References and Recommended Reading}

Papers of particular interest, published recently, have been highlighted as:

- Of importance,

• Of major importance

1. $\quad$ Auger WR, Fedullo PF: Chronic thromboembolic pulmonary hypertension. Sem Resp Crit Care Med 2009, 30:471-483, The authors provide a comprehensive review of CTEPH.

The authors provide a comprehensive review of CTEPH

2. Pengo $\mathrm{V}$, Lensing $\mathrm{A}$, Prins $\mathrm{M}$, et al.: Incidence of chronic thromboembolic pulmonary hypertension after pulmonary embolism. N Engl J Med 2004, 350:2257-2264.

3. Miniati $M$, Simonetta $M$, Bottai $M$, et al.: Survival and restoration of pulmonary perfusion in a longterm follow-up of patients after pulmonary embolism. Medicine 2006, 85:253-262.

4. Jamieson S, Kapelanski D, Sakakibara N, et al.: Pulmonary endarterectomy: experience and lessons learned in 1,500 cases. Ann Thorac Surg 2003, 76:1457-1464.

5. Ribeiro A, Lindmarker P, Johnsson H, et al.: Pulmonary embolism: one-year follow-up with echocardiography Doppler and five-year survival analysis. Circulation 1999, 99:1325-1330.

6. Wolf M, Boyer-Neumann C, Parent F, et al.: Thrombotic risk factors in pulmonary hypertension. Eur Respir J 2000, 15:395-399.

7.• Hoeper MM, Barbera JA, Channick RN, et al.: Diagnosis, assessment, and treatment of nonpulmonary arterial hypertension pulmonary hypertension. J Am Coll Cardiol Suppl 2009, 54:S85-S96,

This is an excellent review of the diagnosis and treatment of patients in pulmonary hypertension groups 2 to 5 of the current PH classification system

8. Thistlethwaite PA, Kaneko K, Madani MM, Jamieson SW: Technique and outcomes of pulmonary endarterectomy surgery. Ann Thorac Cardiovasc Surg 2008, 14:274-282.

9. Bresser P, Pepke-Zaba J, Jais X, et al.: Medical therapies for chronic thromboembolic pulmonary hypertension. An evolving treatment paradigm. Proc Am Thorac Soc 2006, 3:594-600.

10. Jensen K, Kerr K, Fedullo P, et al.: Pulmonary hypertensive medical therapy in chronic thromboembolic pulmonary hypertension before pulmonary thromboendarterectomy. Circulation 2009, 120:1248-1254.

11. Kim N: Assessment of operability in chronic thromboembolic pulmonary hypertension. Proc Am Thorac Soc 2006, 3:589-593.

12. Darteville P, Fadel E, Mussot S, et al.: Chronic thromboembolic pulmonary hypertension. Eur Resp J 2004, 23:637-648.
13. Moser K, Bloor C: Pulmonary vascular lesions occurring in patients with chronic major vessel thromboembolic pulmonary hypertension. Chest 1993, 103:685-692.

14. Reichenberger F, Voswinckel R, Enke B, et al.: Longterm treatment with sildenafil in chronic thromboembolic pulmonary hypertension. Eur Resp J 2007, 30:922-927.

15. Hughes RJ, Jais X, Bonderman D, et al.: The efficacy of bosentan in inoperable chronic thromboembolic pulmonary hypertension : a 1-year follow-up study. Eur Resp J 2006, 28:138-143.

16. Vassallo FG, Kodric M, Scarduelli C, et al.: Bosentan for patients with chronic thromboembolic pulmonary hypertension. Eur J Int Med 2009, 20:24-29.

17. Cabrol S, Souza R, Jais X, et al.: Intravenous epoprostenol in inoperable chronic thromboembolic disease. J Heart Lung Transplant 2007, 26:357-362.

18. Skoro-Sajer N, Bonderman D, Wiesbauer F, et al.: Treprostinil for severe inoperable chronic thromboembolic pulmonary hypertension. J Thromb Haemost 2007, 5:483-489.

19. Krug S, Hammerschmidt S, Pankau H, et al.: Acute improved hemodynamics following inhaled iloprost in chronic thromboembolic pulmonary hypertension. Respiration 2008, 76:154-159.

20.• Jais X, D'Armini A, Jansa P, et al.: Bosentan for treatment of inoperable chronic thromboembolic pulmonary hypertension. J Am Coll Cardiol 2008, 52:2127-2134

This important double-blind, placebo-controlled study examined the efficacy of an ERA in patients with inoperable CTEPH or residual PH post endarterectomy

21. Suntharalingam J, Treacy C, Doughty N, et al.: Longterm use of sildenafil in inoperable chronic thromboembolic pulmonary hypertension. Chest 2008, 134:229-236.

22. Nagaya N, Sasaki N, Ando M, et al.: Prostacyclin therapy before pulmonary thromboendarterectomy in patients with chronic thromboembolic pulmonary hypertension. Chest 2003, 123:338-343.

23. Kramm T, Eberle B, Krummenauer F, et al. Inhaled iloprost in patients with chronic thromboembolic pulmonary hypertension: effects before and after pulmonary thromboendarterectomy. Ann Thorac Surg 2003, 76:711-718.

24. Micromedex. Available at http://www.thomsonhc. com/hcs/librarian. Accessed December 2009. 
25. Condliffe R, Kiely DG, Gibbs SR, et al.: Improved outcomes in medically and surgically treated chronic thromboembolic pulmonary hypertension Am J Resp Crit Care Med 2008, 177:1122-1127,

This article reports on the improving prognosis for CTEPH patients in the United Kingdom as a result of medical and surgical "disease-modifying therapies."

26. Keogh A, Mayer E, Benza R, et al.: Interventional and surgical modalities of treatment in pulmonary hypertension. J Am Coll Cardiol 2009, 54:S67-S77.

27. Hartz R, Byrne J, Levitsky S, et al.: Predictors of mortality in pulmonary thromboendarterectomy. Ann Thorac Surg 1996, 62:1255-1260.

28. Mayer E, Klepetko W: Techniques and outcomes of pulmonary endarterectomy for chronic thromboembolic pulmonary hypertension. Proc Am Thorac Soc 2006, 3:589-593.

29. Iwase T, Nagaya N, Ando M, et al.: Acute and chronic effects of surgical thromboendarterectomy on exercise capacity and ventilatory efficiency in patients with chronic thromboembolic pulmonary hypertension. Heart 2001, 86:188-192.
30. Archibald CJ, Auger WR, Fedullo PF, et al.: Long-term outcome after pulmonary thromboendarterectomy. Am J Respir Crit Care Med 1999, 160:523-528.

31. Corsico AG, D'Armini AM, Cerveri I, et al.: Longterm outcome after pulmonary endarterectomy. Am J Respir Crit Care Med 2008, 178:419-424.

32. Thistlethwaite PA, Madani MM, Kemp AD, et al.: Venovenous extracorporeal life support after pulmonary endarterectomy: indications, techniques, and outcomes. Ann Thorac Surg 2006, 82:2139-2145.

33. Thompson B, Tsui SSL, Dunning J, et al.: Pulmonary endarterectomy is possible and effective without the use of complete circulatory arrest: the UK experience in over 150 patients. Eur J Cardiothorac Surg 2008, 33:157-163.

34. Thistlethwaite PA, Auger WR, Madani MM, et al.: Pulmonary thromboendarterectomy combined with other cardiac operations: indications, surgical approach, and outcome. Ann Thorac Surg 2001, 72:13-19. 